\title{
Bounding the Higgs width at the LHC
}

\section{John M. Campbell}

Fermilab, PO Box 500, Batavia, IL 60510

E-mail: johnmcefnal.gov

\section{R. Keith Ellis*}

Fermilab, PO Box 500, Batavia, IL 60510

E-mail: ellis@fnal.gov

\section{Ciaran Williams}

Niels Bohr International Academy and Discovery Center, The Niels Bohr Institute Blegdamsvej 17, DK-2100 Copenhagen Ø

E-mail: ciaran@nbi.dk

We present results for the Standard model description of the four-lepton production, mediated both by Higgs boson production and by other one-loop standard model processes. The description of four-lepton final states in MCFM v6.8 is reviewed, with special reference to the interference effects that can occur for identical species of leptons. We present results both for interference in the $\ell^{-} \ell^{+} \ell^{-} \ell^{+}$and in the $\ell^{-} \ell^{+} v_{\ell} \bar{v}_{\ell}$ final state. Prospects for further improvement in the theoretical description of of four lepton production are also reviewed.

Loops and Legs in Quantum Field Theory - LL 2014,

27 April - 2 May 2014

Weimar, Germany

\footnotetext{
*Speaker.
} 


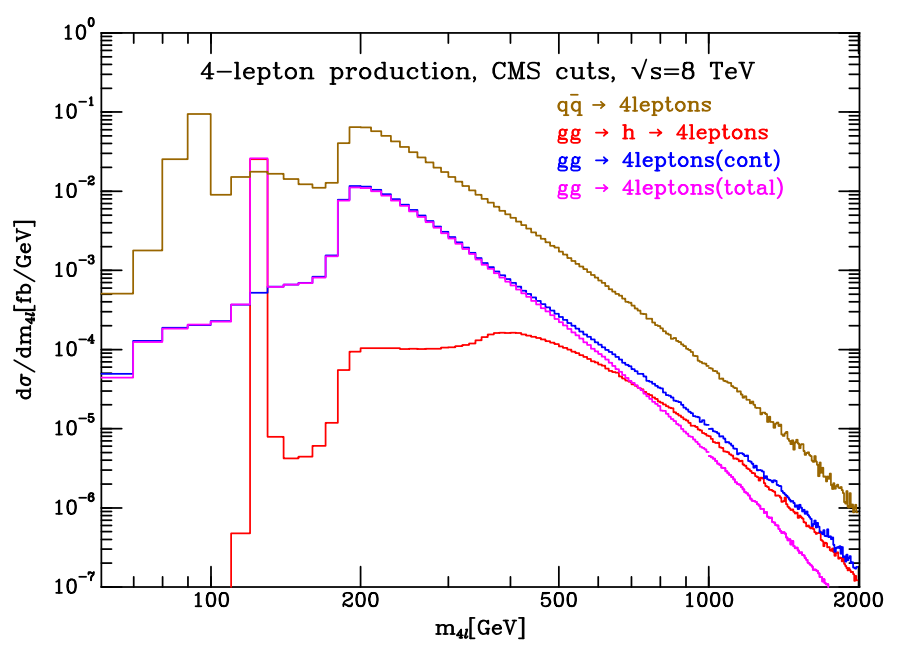

Figure 1: Overall picture at $8 \mathrm{TeV}$, (colour online). In this figure the CMS cuts have been imposed, but the constraint $m_{4 \ell}>100 \mathrm{GeV}$ has been removed to extend the range of the plot.

\section{Off-shell Higgs production}

In an interesting recent paper, Caola and Melnikov [1] proposed to constrain the total width of the Higgs boson using the $H \rightarrow Z Z \rightarrow 4$ lepton-events where the mass of the four leptons, $m_{4 l}$ is away from the Higgs resonance region. This method exploits the fact that at least $15 \%$ of the Higgs cross section, with the Higgs boson decaying to four charged leptons, comes from the off-peak region corresponding to a four-lepton invariant mass above $130 \mathrm{GeV}$ [2]. Various contributions to the four charged lepton final state are shown in Fig. 1. The (red) histogram with the peak at $m_{4 l}=125 \mathrm{GeV}$ is due to the Higgs mediated diagram shown in Fig. 2(a). Considering this diagram alone, the cross section under the Higgs peak is proportional to $g_{i}^{2} g_{f}^{2} / \Gamma_{H}$, where $g_{i}$ is the coupling of the Higgs to the initial state, $g_{f}$ is the coupling to the final state, and $\Gamma_{H}$ is the total Higgs boson width. In contrast the off-shell cross section due to this diagram is independent of the width and proportional to $g_{i}^{2} g_{f}^{2}$. Thus by comparing the measured on-peak/off-peak ratio with the theoretically predicted ratio, one can obtain bounds on the total width of the Higgs boson.

This simple analysis is complicated by a number of factors. First, the contribution to the $g g \rightarrow 4$ lepton final state, shown in Fig. 2(b) ('the background process') interferes with the Higgs production process. This interference is destructive at large $m_{4 \ell}$ as it must be, since the role of the Higgs boson is to cancel the bad high-energy behaviour of the background process. The total rate, resulting from the total amplitude squared, $\left|A^{(a)}+A^{(b)}\right|^{2}$ is shown by the (magenta) curve (the lowest lying curve at large $\left.m_{4 \ell}\right)$ in Fig. 1. Second, the amplitude $\left|A^{(a)}\right|$ contains an enhancement when the two constituents running in the loop are on their mass shells. Observation of this enhancement, which in the standard model occurs at $m_{4 \ell} \sim 2 m_{t}$, would give information about the mass of the particles that couple to the Higgs boson. Third, the $g g$-initiated process has to be isolated from a much bigger $q \bar{q}$ standard model background, represented in Fig 1 by the uppermost (brown) curve. The peak in this histogram at the mass of the $Z$-boson is due to singly resonant diagrams. The curves in Fig 1 are taken from ref. [3] where compact analytic formula for the background process are presented. These formula are implemented into the parton-level integrator MCFM, leading to 


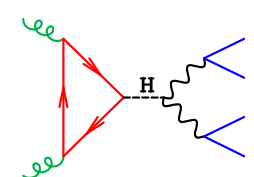

(a)

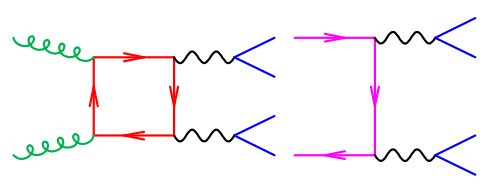

(b)

(c)

Figure 2: Representative diagrams for the partonic processes considered in this paper.

numerically stable leading-order results for the on-peak and off-peak cross sections. The numerical results of ref. [3] correct minor numerical errors in the results of ref. [1].

\section{MCFM: recent progress}

Before commenting on the numerical results for the four lepton final state, we give a brief description of the advances in the description of this process in MCFMv6.8. MCFM is a partonlevel event integrator which gives results for a series of processes, especially those containing the bosons $W, Z$ and $H$ and heavy quarks, $c, b$ and $t$. The new features present in MCFMv6.8 are:-

- Identical particle effects for $Z Z \rightarrow l^{-} l^{+} l^{-} l^{+}$decays;

- Interference effects for $Z Z \rightarrow\left(e^{-} e^{+}\right)\left(v_{e} \bar{v}_{e}\right)$ and $W^{+} W^{-} \rightarrow\left(e^{-} \bar{v}_{e}\right)\left(v_{e} e^{+}\right)$decays;

- Inclusion of triphoton processes $(\gamma \gamma \gamma)[4]$;

- Inclusion of Diphoton + jet processes $(\gamma \gamma+$ jet $)$.

The latter two processes are beyond the scope of this report, but we shall report on our results for the first two.

\subsection{Identical particle effects in $Z Z \rightarrow 4 \ell$}

The original implementation of the four lepton process in MCFM was for distinct leptons, i.e. $e^{-} e^{+} \mu^{-} \mu^{+}$, but this has now been extended to include the case of identical charged leptons. The interference has been included using the same method as in the POWHEG-BOX-V2 [5] to efficiently sample the more complicated phase space.

We use a set of cuts that is very similar to the ones employed in the CMS analysis [6]. In order to assess the effect of the identical particle interference we have set the muon and electron rapidity acceptance to be the same. The detailed cuts are,

$$
\begin{aligned}
& p_{T, \mu}>7 \mathrm{GeV},\left|\eta_{\mu}\right|<2.4, \\
& p_{T, e}>7 \mathrm{GeV},\left|\eta_{e}\right|<2.4 \\
& m_{\ell \ell}>4 \mathrm{GeV}, m_{4 \ell}>100 \mathrm{GeV} .
\end{aligned}
$$

In addition, the transverse momentum of the hardest (next-to-hardest) lepton should be larger than 20 (10) $\mathrm{GeV}$, the invariant mass of the pair of same-flavour leptons closest to the $Z$-mass should be in the interval $40<m_{l l}<120 \mathrm{GeV}$ and the invariant mass of the other pair should be in the 

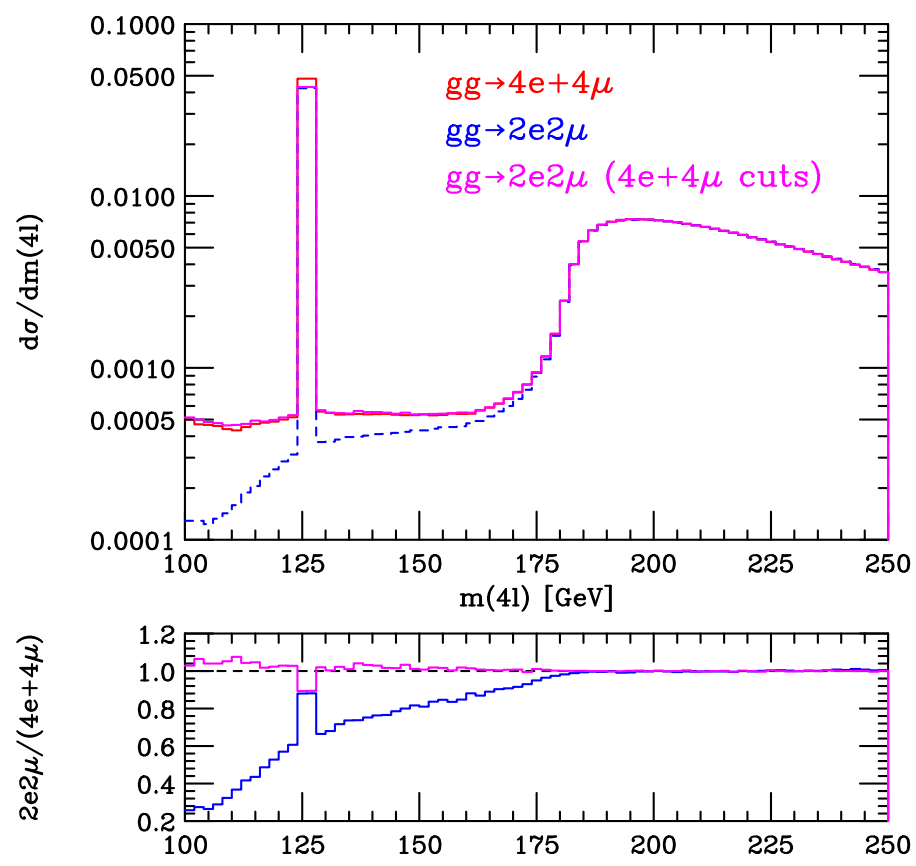

Figure 3: Interference effects in $g g \rightarrow 4 \ell$, including both Higgs and box contributions.

interval $12<m_{l l}<120 \mathrm{GeV}$. We have taken $m_{H}=126 \mathrm{GeV}$ and the QCD renormalization and factorization scales have been set equal to $m_{4 \ell} / 2$. The PDF set used is CT10.

We assess the interference by comparing the $m_{4 \ell}$ spectrum for the $4 e+4 \mu$ final states with the prediction for $2 e 2 \mu$. The overall normalization is then expected to be the same, up to small differences due to the interference. The results are shown at $\sqrt{s}=8 \mathrm{TeV}$ for the complete prediction, combining both Higgs and box diagrams (Fig. 3). For Fig. 3 we have computed the $2 e 2 \mu$ process under two sets of cuts. The first is the physical case, in which we do not attempt to form a $Z$ candidate from lepton pairs of different flavours, i.e. $e^{-} \mu^{+}$and $\mu^{-} e^{+}$. This curve is shown in blue. In the second case we do allow such combinations in order to make a more direct comparison with the $4 e+4 \mu$ case where all leptons have the same flavor. The resulting curve is shown in magenta. Comparing the curves, we see a significant difference between the same- and different-flavor cases solely due to the fact that $Z$-candidates may be improperly assigned in the same-flavor case.

In the bin that contains the Higgs resonance there is a significant difference between the $4 e+$ $4 \mu$ and $2 e 2 \mu$ predictions. There the effect of the interference is well-known [7] and, for these cuts, it results in a $13 \%$ higher cross section for identical leptons. There is essentially no effect from the interference in the region $m_{4 \ell}>2 m_{Z}$.

\subsection{Interference effects for the $2 \ell 2 v$ final state}

For this final state, interference can occur between final states produced by leptonic decays of a $W W\left(\rightarrow v_{l} l^{+} l^{-} \bar{v}_{l}\right)$ and $Z Z\left(\rightarrow l^{-} l^{+} v_{l} \bar{v}_{l}\right)$. It has been included using the same method as in gg2VV [8] to efficiently sample the more complicated phase space.

We use a set of cuts that is very similar to the ones employed in the CMS analysis [9, 10]. In 

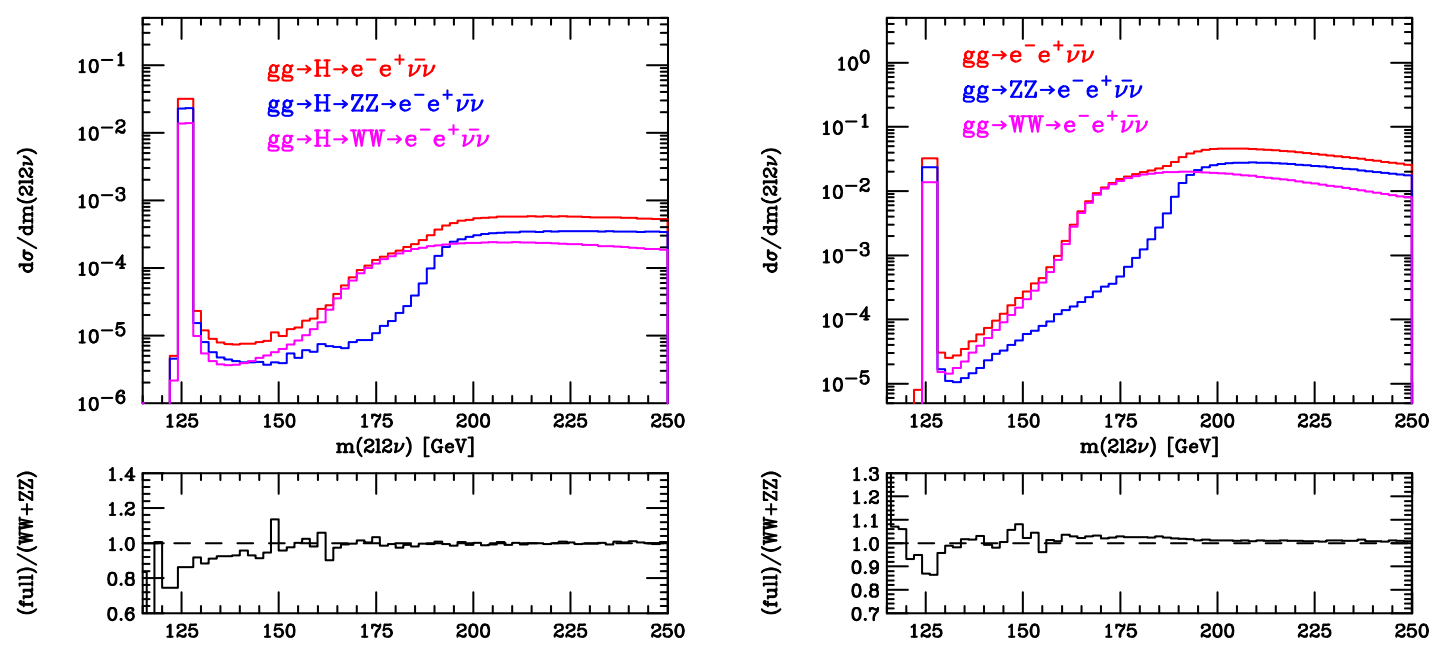

Figure 4: Interference effects in $g g \rightarrow H \rightarrow 2 \ell 2 v$. Figure 5: Interference effects in $g g \rightarrow 2 \ell 2 v$, including both Higgs and box contributions.

order to better explore the effects of the interference we have relaxed the missing $E_{T}$ cut to allow for some Higgs contribution (the original analysis is aimed at a heavy Higgs bosons).

$$
\begin{gathered}
p_{T, e}>20 \mathrm{GeV}, \quad\left|\eta_{e}\right|<2.4 \\
\left|m_{\ell \ell}-m_{Z}\right|<15 \mathrm{GeV}, \quad M E T>25 \mathrm{GeV} .
\end{gathered}
$$

The discussion below uses $m_{4 \ell}$ for simplicity, even though it is not directly observable in the experiment. We assess the interference by comparing the $m_{4 \ell}$ spectrum for the sum of the $W W$ and $Z Z$ final states with the prediction obtained by summing amplitudes before squaring. We show results for $e^{-} e^{+}$and a sum over all three neutrinos. Note that the interference only enters for the one final state $e^{-} e^{+} v_{e} \bar{v}_{e}$. Results are shown at $\sqrt{s}=8 \mathrm{TeV}$ for the Higgs amplitudes alone (Fig. 4) and for the complete prediction, combining both Higgs and box diagrams (Fig. 5). As can be seen from the lower panes in the two figures, there is no effect from the interference in the region $m_{4 \ell}>2 m_{Z}$. In contrast, there is a signficant effect below the threshold for production of two real $Z$ bosons. Since the cross section is very small for the continuum diagrams alone, this is not phenomenologically relevant except in the bin that contains the Higgs resonance. There, the effect of the interference is negative [8]. For these cuts, it results in a 25\% lower cross section for the Higgs diagrams alone and a $14 \%$ lower cross section when all diagrams are considered.

\section{Theoretical and experimental results in off-peak four lepton production.}

The expected cross section in the off-shell region due to the signal and signal-background interference is presented in ref. [3]. The number of such events expected in the combined 7 and $8 \mathrm{TeV}$ data sample is obtained by summing the appropriately-weighted cross sections and normalizing to the peak cross section reported in Ref. [9]. We find,

$$
N_{o f f}^{4 \ell}\left(m_{4 \ell}>130 \mathrm{GeV}\right)=2.78\left(\frac{\Gamma_{H}}{\Gamma_{H}^{S M}}\right)-5.95 \sqrt{\frac{\Gamma_{H}}{\Gamma_{H}^{S M}}}
$$




$$
N_{o f f}^{4 \ell}\left(m_{4 \ell}>300 \mathrm{GeV}\right)=2.02\left(\frac{\Gamma_{H}}{\Gamma_{H}^{S M}}\right)-2.91 \sqrt{\frac{\Gamma_{H}}{\Gamma_{H}^{S M}}}
$$

The linear term is due to the Higgs contribution, whereas the square root contribution is due to Higgs-box diagram interference. By setting $\Gamma_{H}$ to its standard model value we see that the interference is large and negative. Using the matrix elements of ref. [3] the CMS collaboration has performed a maximum likelihood fit to the on-peak and off-peak regions and obtain [6] an upper limit on the Higgs boson width of $\Gamma_{H}<22 \mathrm{MeV}$ at a 95\% confidence level, which is 5.4 times the expected standard model value.

\subsection{Theoretical errors}

The theoretical estimates presented so far for the above-peak production mediated by Higgs boson diagrams, are based on a one-loop calculation, which in this case constitutes a leading order calculation. We have detailed information about the higher order corrections to the signal process, $g g \rightarrow H \rightarrow Z Z$. It is known that NLO corrections increase the lowest order prediction by about $80-100 \%$. The NNLO prediction, computed in the infinite top mass limit, increases the cross section by a further $25 \%[11,12,13]$. Information about the influence of higher order corrections to the background process $g g \rightarrow Z Z$ is much scarcer. In this situation the CMS collaboration [14] assign a $K$-factor equal to the one used for the signal to the $g g \rightarrow Z Z$ background. They further assign a $10 \%$ theoretical systematic error to this assumption.

However if we allow a relative $K$-factor between the Higgs diagrams, for which the $K$-factor is known to be $>2$ and the continuum diagrams for which the $K$-factor is not known, the limit, assuming a bound on the number of excess events at 4.1 , is

$$
\frac{\Gamma_{H}}{\Gamma_{H}^{S M}}<\{4.1,5.4,7.1\} \text { for } \sqrt{K}=\{0.7,1.0,1.3\}
$$

The dependence of the assumed limit on the relative $K$-factor is shown in Fig. 6 .

Reference [15] estimates the $K$-factor for the continuum process $g g \rightarrow W^{+} W^{-}$using the soft collinear approximation. This should be particularly appropriate for the case of Heavy Higgs boson, considered in ref. [15]. Although the full soft approximation is not known, the missing piece can be estimated using the results for $g g \rightarrow H H$ which is known [16] in the heavy top limit. This argument exploits the fact that if the vector boson production is dominated by longitudinal modes, the equivalence theorem should allow the missing soft term to be calculated.

Figure 7 shows the contribution from the various quarks to the Higgs-Box interference. It is only above $m_{4 \ell}>400 \mathrm{GeV}$ that the top loop (and hence the longitudinal modes) can be said to dominate. Therefore is is only in this region that the argument of ref. [15] can be strictly applied. Moreover the discussion of ref. [15] refers to a heavy Higgs boson for which the interference is small and positive, whereas for the physical Higgs boson the interference is large and negative. It is therefore our opinion that the assertion that the relative $K$-factor between signal and background is small, and therefore that the theoretical error is as small as $10 \%$ (as used in the experimental paper [6]) is not yet established. In the circumstances it is appropriate to perform a full NLO calculation of this interference. 


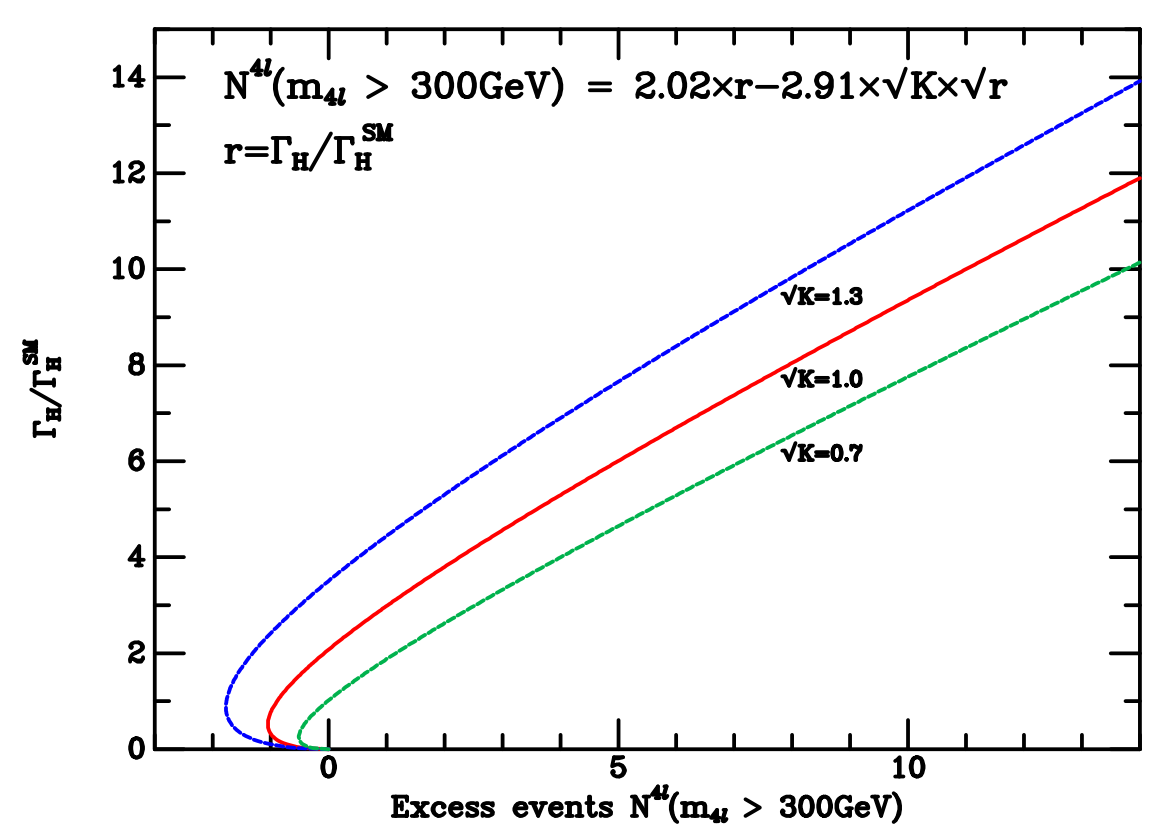

Figure 6: Influence of relative K-factor on extracted limit for $\Gamma_{H} / \Gamma_{H}^{S M}$ as a function of the number of excess events from $g g$-induced Higgs boson related processes.

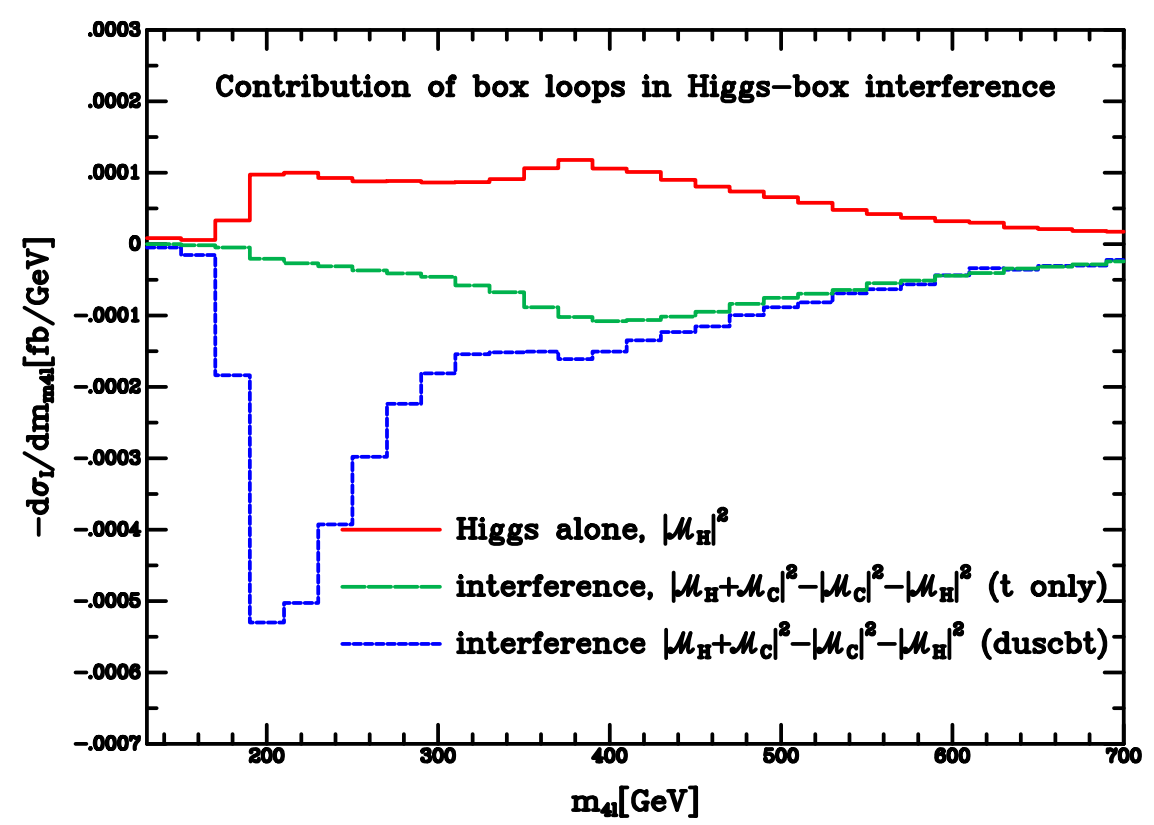

Figure 7: Contribution of the various box loops to the interference. 


\section{References}

[1] F. Caola, K. Melnikov, Constraining the Higgs boson width with ZZ production at the LHC, Phys. Rev. D88 (2013) 054024. arXiv:1307.4935, doi:10.1103/PhysRevD.88.054024.

[2] N. Kauer, G. Passarino, Inadequacy of zero-width approximation for a light Higgs boson signal, JHEP 1208 (2012) 116. arXiv:1206.4803, doi:10.1007/JHEP08(2012)116.

[3] J. M. Campbell, R. K. Ellis, C. Williams, Bounding the Higgs width at the LHC using full analytic results for $g g \rightarrow e^{-} e^{+} \mu^{-} \mu^{+}$, JHEP 1404 (2014) 060. arXiv:1311.3589, doi:10.1007/JHEP04(2014)060.

[4] J. M. Campbell, C. Williams, Triphoton production at hadron colliders, Phys. Rev. D89 (2014) 113001. arXiv:1403.2641, doi:10.1103/PhysRevD.89.113001.

[5] P. Nason, G. Zanderighi, $W^{+} W^{-}, W Z$ and $Z Z$ production in the POWHEG-BOX-V2, Eur. Phys. J. C74 (2014) 2702. arXiv:1311.1365, doi:10.1140/epjc/s10052-013-2702-5.

[6] V. Khachatryan, et al., Constraints on the Higgs boson width from off-shell production and decay to Z-boson pairs arXiv:1405.3455.

[7] S. Heinemeyer, et al., Handbook of LHC Higgs Cross Sections: 3. Higgs Properties arXiv:1307.1347, doi:10.5170/CERN-2013-004.

[8] N. Kauer, Interference effects for $H \rightarrow W W / Z Z \rightarrow \ell \bar{v}_{\ell} \bar{\ell} v_{\ell}$ searches in gluon fusion at the LHC, JHEP 1312 (2013) 082. arXiv:1310.7011, doi:10.1007/JHEP12(2013)082.

[9] Properties of the Higgs-like boson in the decay $H \rightarrow Z Z \rightarrow 4 l$ in pp collisions at $\sqrt{s}=7$ and $8 \mathrm{TeV}$, CMS Collaboration, CMS-PAS-HIG-13-002 (2013).

[10] Search for a heavy Higgs boson in the $H \rightarrow Z Z \rightarrow 2 l 2 v$ channel in pp collisions at $\sqrt{(s)}=7$ and 8 TeV, CMS Collaboration, CMS-PAS-HIG-13-014 (2013).

[11] V. Ravindran, J. Smith, W. L. van Neerven, NNLO corrections to the total cross-section for Higgs boson production in hadron hadron collisions, Nucl. Phys. B665 (2003) 325-366. arXiv:hep-ph/0302135, doi:10.1016/S0550-3213(03)00457-7.

[12] R. V. Harlander, W. B. Kilgore, Next-to-next-to-leading order Higgs production at hadron colliders, Phys. Rev. Lett. 88 (2002) 201801. arXiv:hep-ph/0201206, doi:10.1103/PhysRevLett.88.201801.

[13] S. Dittmaier, et al., Handbook of LHC Higgs Cross Sections: 1. Inclusive Observables arXiv:1101.0593, doi:10.5170/CERN-2011-002.

[14] Constraints on the Higgs boson width from off-shell production and decay to $Z Z$ to $l l l l$ and $l l v v$, CMS Collaboration, CMS-PAS-HIG-14-002 (2014).

[15] M. Bonvini, F. Caola, S. Forte, K. Melnikov, G. Ridolfi, Signal-background interference effects for $g g H W^{+} W^{-}$beyond leading order, Phys. Rev. D88 (3) (2013) 034032. arXiv:1304.3053, doi:10.1103/PhysRevD.88.034032.

[16] S. Dawson, S. Dittmaier, M. Spira, Neutral Higgs boson pair production at hadron colliders: QCD corrections, Phys. Rev. D58 (1998) 115012. arXiv:hep-ph/9805244, doi:10.1103/PhysRevD.58.115012. 